\title{
Paeoniflorin prevents hypoxia-induced epithelial- mesenchymal transition in human breast cancer cells
}

This article was published in the following Dove Press journal:

OncoTargets and Therapy

27 April 2016

Number of times this article has been viewed

\section{Zhenyu Zhou',* \\ Shunchang Wang ${ }^{1, *}$ \\ Caijuan Song ${ }^{2}$ \\ Zhuang $\mathrm{Hu}^{\prime}$}

'Department of Thyroid and Breast, Huaihe Hospital, Henan University, Kaifeng, ${ }^{2}$ Department of Immunization Program, Zhengzhou Center for Disease Control and Prevention, Zhengzhou, People's Republic of China

*These authors contributed equally to this work
Correspondence: Zhenyu Zhou Department of Thyroid and Breast, Huaihe Hospital, Henan University, No 8 Baobei Road, Kaifeng 475000, People's Republic of China Tel/fax +86 37I 23906534 Email zhythyr@163.com

\begin{abstract}
Paeoniflorin (PF) is a monoterpene glycoside extracted from the root of Paeonia lactiflora Pall. Previous studies have demonstrated that PF inhibits the growth, invasion, and metastasis of tumors in vivo and in vitro. However, the effect of PF on hypoxia-induced epithelialmesenchymal transition (EMT) in breast cancer cells remains unknown. Therefore, the objective of this study was to investigate the effect of PF on hypoxia-induced EMT in breast cancer cells, as well as characterize the underlying mechanism. The results presented in this study demonstrate that PF blocks the migration and invasion of breast cancer cells by repressing EMT under hypoxic conditions. PF also significantly attenuated the hypoxia-induced increase in HIF-1 $\alpha$ level. Furthermore, PF prevented hypoxia-induced expression of phosphorylated PI3K and Akt in MDA-MB-231 cells. In conclusion, PF prevented hypoxia-induced EMT in breast cancer cells by inhibiting HIF-1 $\alpha$ expression via modulation of PI3K/Akt signaling pathway. This finding provides evidence that PF can serve as a therapeutic agent for the treatment of breast cancer.
\end{abstract}

Keywords: paeoniflorin, breast cancer, hypoxia, epithelial-mesenchymal transition, PI3K/ Akt signaling pathway

\section{Introduction}

Breast cancer is the leading malignancy in females worldwide and the incidence rates have been increasing annually. ${ }^{1}$ Although various treatments for breast cancer have been used and have been improved recently, the clinical outcome of patients remains unsatisfactory. ${ }^{2-4}$ Thus, development of effective chemotherapeutic adjuvants is important to increase cure rates in these patients.

Epithelial-mesenchymal transition (EMT) is a crucial step in tumor progression and has an important role during cancer invasion and metastasis. ${ }^{5-7}$ Mounting evidence shows that the hypoxic environment in most of the rapidly growing solid tumors results from the poor development of angiogenic vessels which leads to insufficient supply of oxygen. ${ }^{8,9}$ It was demonstrated that hypoxia might stimulate EMT in breast cancer cells. Hypoxia increased the expression of Slug and Snail in breast cancer cells, which in turn inhibited E-cadherin expression. ${ }^{10,11}$ Therefore, inhibiting hypoxia-induced EMT can be used as a promising target for developing new and effective anticancer therapy.

The root of Paeonia lactiflora Pall. (family Ranunculaceae) has long been used in oriental medicine for pain, muscle spasm, inflammation, menstrual dysfunction, and degenerative disorders. Paeoniflorin (PF) is a monoterpene glycoside extracted from the root of $P$. lactiflora Pall. Increasing evidences have reported that $\mathrm{PF}$ possesses a variety of physiological properties, including immune regulation, anti-inflammation, and 
protection from myocardial infarction. ${ }^{12-14}$ Furthermore, PF has been reported to inhibit the growth, invasion, and metastasis of tumors in vivo and in vitro. ${ }^{15-18}$ However, the effect of PF on hypoxia-induced EMT in breast cancer cells remains unknown. Therefore, the objective of this study was to investigate the effect of PF on hypoxia-induced EMT in breast cancer cells, as well as characterize the underlying mechanism.

\section{Materials and methods}

Reagents

PF (99\% purity) was obtained from Nanjing Institute for Drug Control (Nanjing, People's Republic of China). The anti-Ecadherin, anti-cadherin, anti-vimentin, anti-HIF-1 $\alpha$, antiPI3K, anti-p-PI3K, anti-Akt, anti-p-Akt, and anti-GAPDH antibodies were purchased from Santa Cruz Biotechnology Inc., (Dallas, TX, USA). All other chemicals and reagents were purchased from Sigma-Aldrich Co., (St Louis, MO, USA).

\section{Cell lines and cell culture conditions}

Human breast cancer cell lines (MDA-MB-231 and MCF-7) were purchased from the American Type Culture Collection (ATCC, Manassas, VA, USA) and maintained in Dulbecco's Modified Eagle's Medium (DMEM) with 10\% fetal bovine serum (FBS), 2 mmol/L L-glutamine, 100 units/mL penicillin, and $100 \mu \mathrm{g} / \mathrm{mL}$ streptomycin (Sigma-Aldrich Co.) in a humidified $5 \% \mathrm{CO}_{2}$ atmosphere at $37^{\circ} \mathrm{C}$. The protocol and procedure of the experiment were approved by the Ethics Committee of Huaihe Hospital.

For hypoxic conditions, cells were seeded in a six-well plate at a density of $1 \times 10^{5}$ cells/well and cultured in hypoxic conditions $\left(5 \% \mathrm{CO}_{2}\right.$ and $\left.1 \% \mathrm{O}_{2}[\mathrm{v} / \mathrm{v}]\right)$, balanced with $\mathrm{N}_{2}$ gas, at $37^{\circ} \mathrm{C}$ for 12,24 , and 48 hours. For normoxic conditions, cells were exposed to normoxic $\left(21 \% \mathrm{O}_{2}\right.$, at room temperature) conditions. The medium was then replaced with new medium supplemented with various concentrations of PF (12.5-50 $\mu \mathrm{M})$.

\section{Determination of cell viability}

The number of viable cells was determined by the trypan blue exclusion assay. In brief, MDA-MB-231 and MCF-7 cells were seeded at a density of $1 \times 10^{5}$ cells/well in six-well plates. After reaching a confluency of $90 \%$, the cells were cultured in serum-free medium for 24 hours and then stimulated with various concentrations of PF (12.5-50 $\mu \mathrm{M})$ for 24 hours. Only dimethyl sulfoxide (DMSO) was added for the control group. After trypsinization, the cells were stained with $40 \mu \mathrm{L}$ trypan blue (Sigma-Aldrich Co.). The number of viable cells was determined using a hemocytometer (Abcam, Cambridge, UK).

\section{Cell migration and invasion assays}

In vitro transwell migration assay was performed in modified Boyden chambers with $8 \mathrm{~mm}$ pore filter inserts in 24-well plates (BD Biosciences, San Jose, CA, USA). Briefly, MDAMB-231 and MCF-7 cells $\left(1 \times 10^{5}\right.$ cells/well) were harvested and suspended in serum-free Roswell Park Memorial Institute (RPMI) 1640 medium. After that, cancer cells were plated into the top chamber of the transwell chambers. The lower chamber was filled with RPMI 1640 medium containing 10\% FBS. After 24 hours, cells on the upper membrane surface were removed with cotton-tipped swabs, and those that had migrated to the bottom side of the filter were subsequently fixed in methanol for 15 minutes and stained with $0.05 \%$ crystal violet in phosphate-buffered saline for 15 minutes. The number of cells per four high-power fields was counted under a microscope in order to determine the average number of cells that had migrated.

The invasion assay was performed using BD BioCoat Matrigel invasion chambers (BD Biosciences; $8 \mathrm{~mm}$ pore size). The same procedures as described earlier were used, except that the filters were precoated with $100 \mathrm{~mL}$ Matrigel.

\section{Real-time quantitative polymerase chain reaction analysis}

Total RNA from breast cancer cells was prepared with Trizol (Thermo Fisher Scientific, Waltham, MA, USA) according to the manufacturer's protocol. Then, cDNA was synthesized using a two-step reverse transcription reaction kit (Takara, Dalian, People's Republic of China). The levels of $H I F-1 \alpha$ gene mRNA transcripts were analyzed by using specific primers and SYBR Green I reagent and the real-time polymerase chain reaction kit, according to the manufacturer's instructions, using the Bio-Rad iQ5 Quantitative PCR System (Takara). The specific primers for HIF-1 $\alpha$ were forward: 5'-AGCCGAGGAAGAACTATGAAC-3', reverse: 5'-ATTTGATGGGTGAGGAATGGG- $3^{\prime}$ and $\beta$-actin were forward: 5'-GAGCTACGAGCTGCCTGACG-3', reverse: 5'-CCTAGAAGCATTTGCGGTGG-3'. The cycling conditions included a holding step at $95^{\circ} \mathrm{C}$ for 10 minutes, and 35 cycles at $95^{\circ} \mathrm{C}$ for 25 seconds, $59^{\circ} \mathrm{C}$ for 30 seconds, and $69^{\circ} \mathrm{C}$ for 30 seconds. Quantitative real-time PCR analysis was performed using an ABI 7500 Sequence Detector (ABI, Warrington, UK), and the results were analyzed by the method of $2^{-\Delta \Delta \mathrm{ct}}$.

\section{Western blotting}

Total protein was extracted from breast cancer cells using radioimmunoprecipitation assay (RIPA) lysis buffer (Beyotime, Nantong, People's Republic of China) according 
to the manufacturer's instructions. Equal amounts of protein $(30 \mu \mathrm{g})$ were separated by sodium dodecyl sulfate polyacrylamide gel electrophoresis and transferred onto polyvinylidene difluoride membranes (Whatman Schleicher \& Schuell, Middlesex, UK). After blocking in Tris-buffered saline (TBS) buffer $(50 \mathrm{mmol} / \mathrm{L} \mathrm{NaCl}, 10 \mathrm{mmol} / \mathrm{L}$ Tris, pH 7.4) containing $5 \%$ nonfat milk, the membrane was immunoblotted with primary antibodies (anti-E-cadherin, anti-N-cadherin, anti-vimentin, anti-HIF-1 $\alpha$, anti-PI3K, anti-p-PI3K, anti-Akt, anti-p-Akt, and anti-GAPDH antibodies; Santa Cruz Biotechnology Inc.) overnight at $4^{\circ} \mathrm{C}$, followed by three washes in TBS with Tween-20 (TBST) for 10 minutes. Subsequently, the membranes were incubated with horseradish peroxidase-conjugated secondary antibody for 1 hour at room temperature. After washing, the sites of antibody binding were visualized by chemiluminescence (Boehringer Mannheim, Mannheim, Germany).

\section{siRNA knockdown of HIF-I $\alpha$}

MDA-MB-231 cells were transfected with siRNA against HIF- $1 \alpha$ and scramble siRNA (negative control) using
LipofectamineTM2000 (Thermo Fisher Scientific), according to the manufacturer's protocols. Following transfection, cells were incubated at $37^{\circ} \mathrm{C}$ in a $\mathrm{CO}_{2}$ incubator for 24 hours before being harvested for the assays described earlier.

\section{Statistical analysis}

All results were reported as mean \pm standard deviation (SD). The differences between groups were compared by Dunnett's test subsequent to analysis of variance (ANOVA). $P<0.05$ was considered to be significant.

\section{Results}

\section{PF inhibits the viability of breast cancer cells cultured in hypoxia}

First, we determined the effect of hypoxia on cell viability. As shown in Figure 1A, the cell viability was not significantly affected with an exposure to hypoxia for 24 hours, while the viability was decreased by $28.2 \% \pm 3.1 \%$ after treatment with hypoxia for 48 hours. Thus, MDA-MB-231 cells were exposed to hypoxia for 24 hours in the subsequent experiments. Next, to examine the effect of PF on cell viability under hypoxia
A

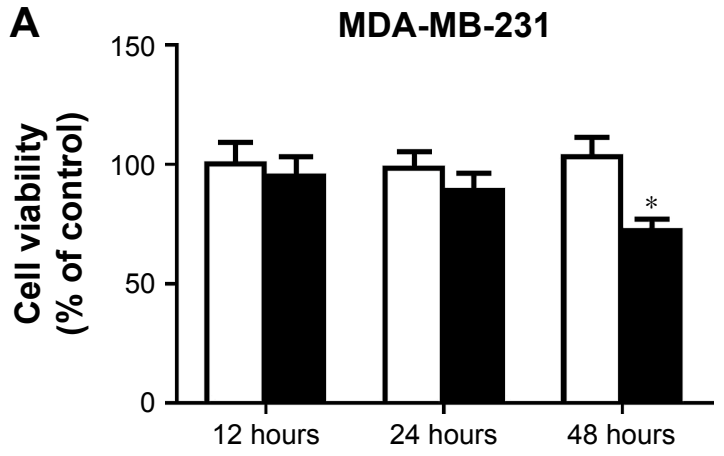

C

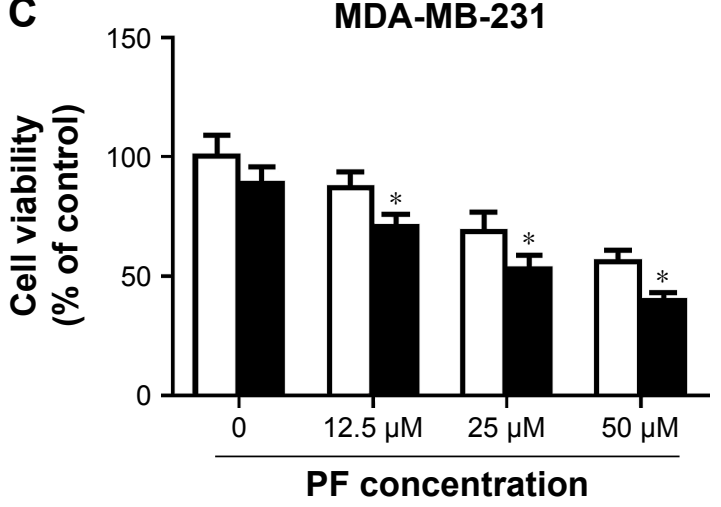

B

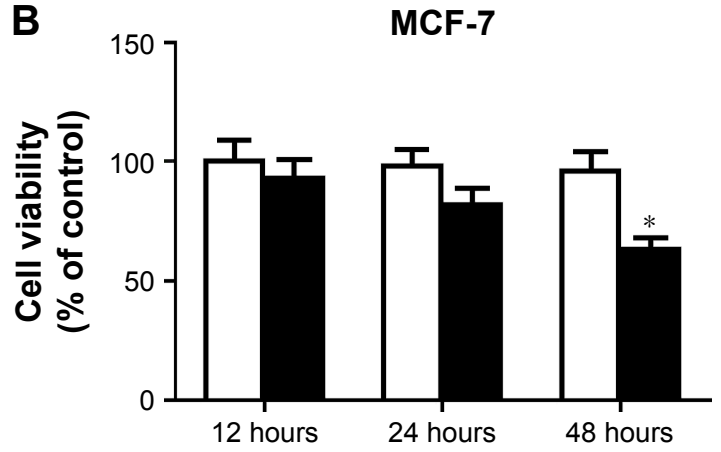

D

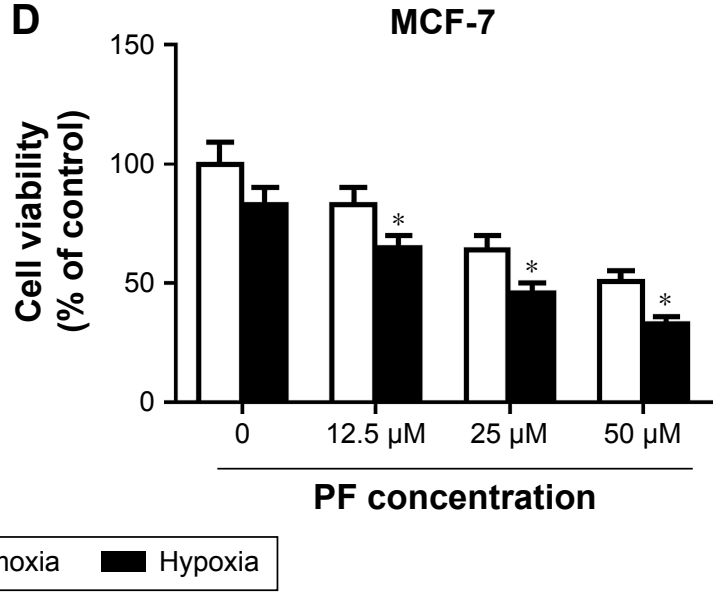

Figure I PF inhibits the viability of breast cancer cells cultured in hypoxia.

Notes: (A, B) MDA-MB-23I and MCF-7 cells were grown under normoxia and hypoxia for 12, 24, and 48 hours. Cell proliferation was evaluated by the trypan blue exclusion assay. (C, D) MDA-MB-23I and MCF-7 cells grown under normoxia or hypoxia were treated with different doses of PF for 24 hours. Cell proliferation was evaluated by the trypan blue exclusion assay. Each bar represents the mean $\pm S D$. The results were reproduced in three independent experiments. $* P<0.05$ vs normoxia group.

Abbreviations: PF, paeoniflorin; SD, standard deviation. 
conditions, MDA-MB-231 cells were treated with PF and incubated under normoxic and hypoxic conditions for 24 hours. As shown in Figure 1C, the inhibitory effect of PF on viability was significantly higher on MDA-MB-231 cells grown under hypoxia than those cultured under normoxia. Similar results were observed in MCF-7 cells (Figure 1B and D).

\section{PF prevented the hypoxia-induced migration and invasion of breast cancer cells}

Hypoxia has been reported to induce invasion and migration of cancer cells. So, we examined the effect of PF on hypoxiainduced cell migration and invasion. As shown in Figure 2A, compared with untreated MDA-MB-231 cells, hypoxia significantly increased the number of cells migrating in Boyden chambers. However, the hypoxia-induced migration of MDA-MB-231 cells decreased by $29 \%$ ( $P<0.05$ vs hypoxia controls) in PF-treated cells. Similarly, hypoxic PF-treated cells showed a $31 \%$ reduction $(P<0.05$ vs hypoxia controls) in the invasive capacity of MDA-MB-231 cells compared to the hypoxia-only controls (Figure 2B). In addition, we also found that $\mathrm{PF}$ prevented the hypoxia-induced migration and invasion of MCF-7 cells (Figure 2C and D).

A

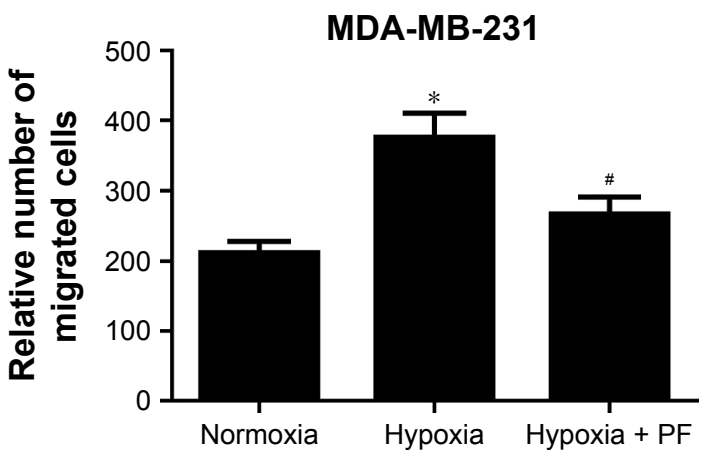

C

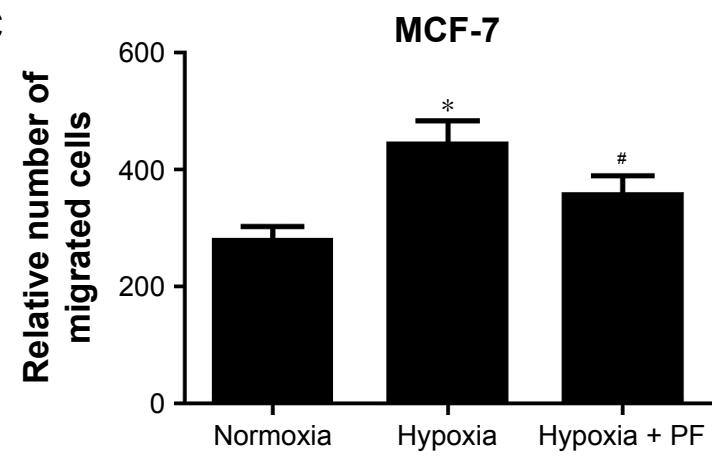

PF prevented the hypoxia-induced EMT in breast cancer cells

Then, we investigated the effect of PF on hypoxia-induced EMT in breast cancer cells. As shown in Figure 3A, hypoxia treatment significantly decreased the expression of E-cadherin and increased the expression of $\mathrm{N}$-cadherin and vimentin in MDA-MB-231 cells. Moreover, PF-treated MDA-MB-231 cells cultured under hypoxic conditions showed that E-cadherin was recovered by $44 \%$, whereas $\mathrm{N}$-cadherin and vimentin was reduced by $33 \%$ and $47 \%$, respectively. Similar results were found in MCF-7 cells (Figure 3B).

\section{HIF-I $\alpha$ is involved in the prevention of hypoxia-induced EMT by PF}

HIF-1 is the best regulator under hypoxia exposure and is involved in the process of EMT. So, we investigated whether the inhibiting effect of PF against hypoxia-induced EMT involves modulation of the HIF-1 $\alpha$ expression. As shown in Figure 4A, PF treatment suppressed the hypoxiainduced HIF- $1 \alpha$ expression. To further verify the role of HIF- $1 \alpha$ in hypoxia-mediated EMT, a lentivirus-mediated siRNA-HIF-1 $\alpha$ was designed and transfected into the MDA-MB-231 cells. As shown in Figure 4B, knockdown
B

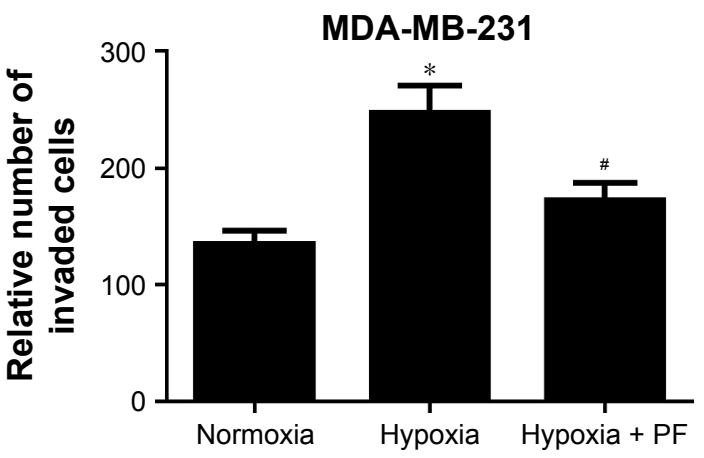

D

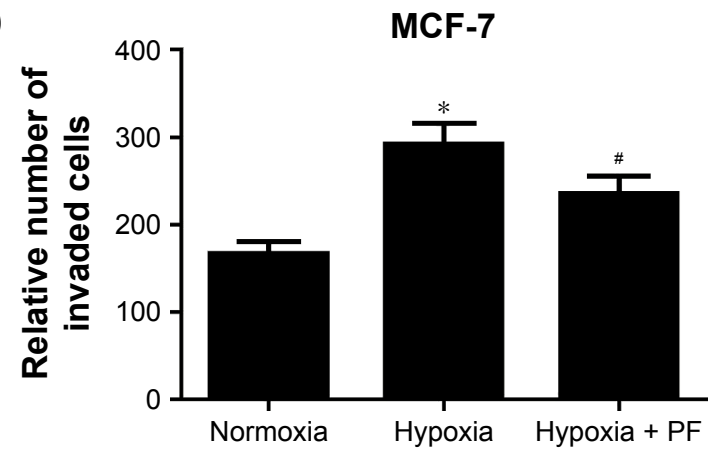

Figure $2 \mathrm{PF}$ prevented the hypoxia-induced migration and invasion of breast cancer cells.

Notes: MDA-MB-23I and MCF-7 cells grown under normoxia or hypoxia were treated with $25 \mu \mathrm{M}$ PF for 24 hours. (A, B) The migration and invasion of MDA-MB-23I cells were assessed by transwell assays. (C, D) The migration and invasion of MCF-7 cells were assessed by transwell assays. Each bar represents the mean \pm SD. The results were reproduced in three independent experiments. ${ }^{*} P<0.05$ vs normoxia group, ${ }^{\#} P<0.05$ vs hypoxia group.

Abbreviations: PF, paeoniflorin; SD, standard deviation. 
A

MDA-MB-231

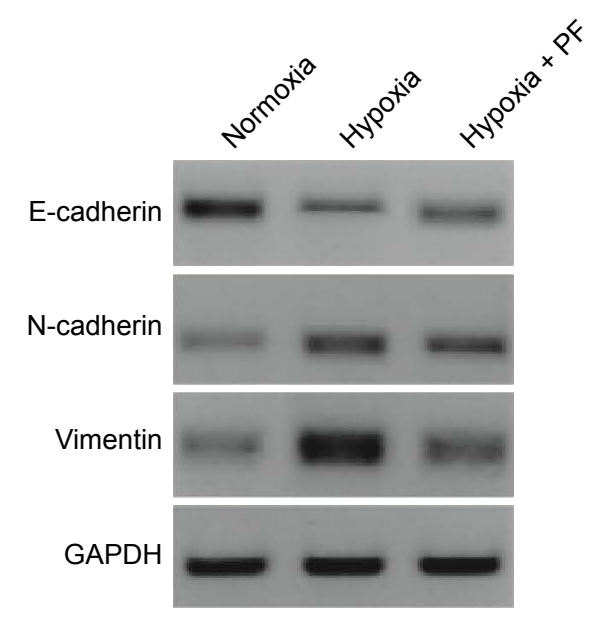

B

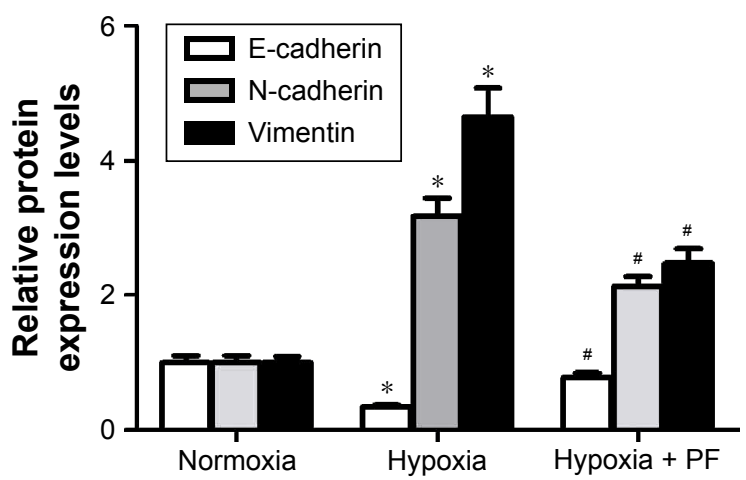

MCF-7
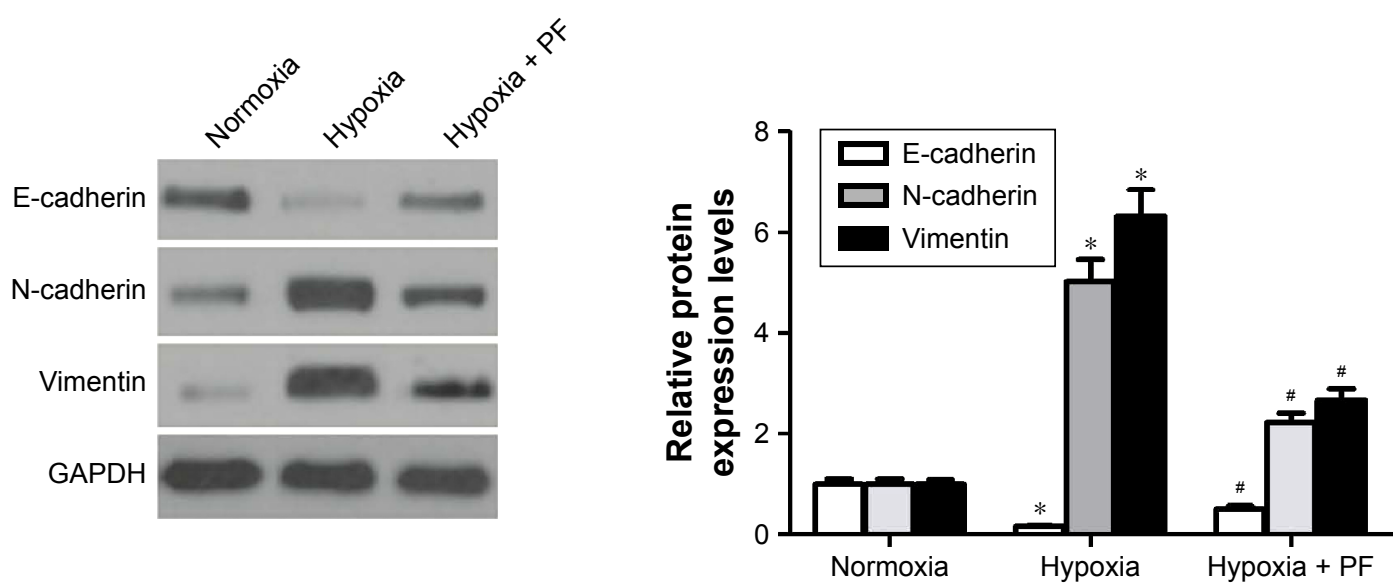

Figure $3 \mathrm{PF}$ prevented the hypoxia-induced EMT in breast cancer cells.

Notes: (A) MDA-MB-23I cells grown under normoxia and hypoxia were treated with $25 \mu \mathrm{M}$ PF for 24 hours. E-cadherin, N-cadherin, and vimentin expressions were assessed by Western blot analysis. The relative protein expression levels of E-cadherin, N-cadherin, and vimentin were quantified using Image-Pro Plus 6.0 software (Media Cybernetics, Silver Spring, MD, USA) and normalized to GAPDH. (B) MCF-7 cells grown under normoxia and hypoxia were treated with $25 \mu \mathrm{M}$ PF for 24 hours. E-cadherin, $\mathrm{N}$-cadherin, and vimentin expressions were assessed by Western blot analysis. The relative protein expression levels of E-cadherin, $\mathrm{N}$-cadherin, and vimentin were quantified using Image-Pro Plus 6.0 software and normalized to GAPDH. Each bar represents the mean $\pm \mathrm{SD}$. The results were reproduced in three independent experiments. $* P<0.05$ vs normoxia group, ${ }^{\sharp}<<0.05$ vs hypoxia group.

Abbreviations: PF, paeoniflorin; EMT, epithelial-mesenchymal transition; SD, standard deviation.

of HIF-1 $\alpha$ significantly inhibited hypoxia-induced HIF-1 $\alpha$ expression. Moreover, knockdown of HIF-1 $\alpha$ obviously restored hypoxia-induced reduction of E-cadherin expression in MDA-MB-231 cells (Figure 4C).

\section{PF-mediated blockade of HIF-I $\alpha$ and EMT involved PI3K/Akt pathway}

Previous studies showed that PI3K/Akt signaling controls the levels of HIF-1 $\alpha .^{19,20}$ Therefore, we postulated that PF might be involved in the regulation of HIF-1 $\alpha$ expression via inhibiting PI3K/Akt signaling. As shown in Figure 5, hypoxia treatment significantly increased the expression of phosphorylated PI3K and Akt in MDA-MB-231 cells.
When treated with PF, the increase in levels of phosphorylated PI3K and Akt was reversed, whereas total levels of $\mathrm{PI} 3 \mathrm{~K}$ and Akt remained unaffected.

\section{Discussion}

The results presented in this study demonstrate that PF blocks the migration and invasion of breast cancer cells by repressing EMT under hypoxic conditions. PF also significantly attenuated the hypoxia-induced increase of HIF-1 $\alpha$ level. Furthermore, PF prevented the hypoxia-induced expression of phosphorylated PI3K and Akt in breast cancer cells.

$\mathrm{PF}$ is reported to have antitumor effect. Hung et al reported that PF inhibited the proliferation of non-small-cell 
A
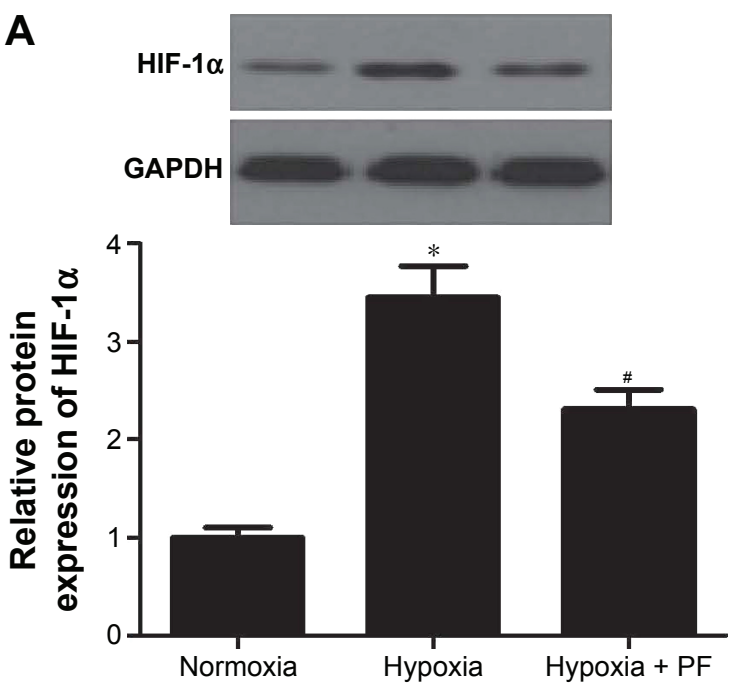

B
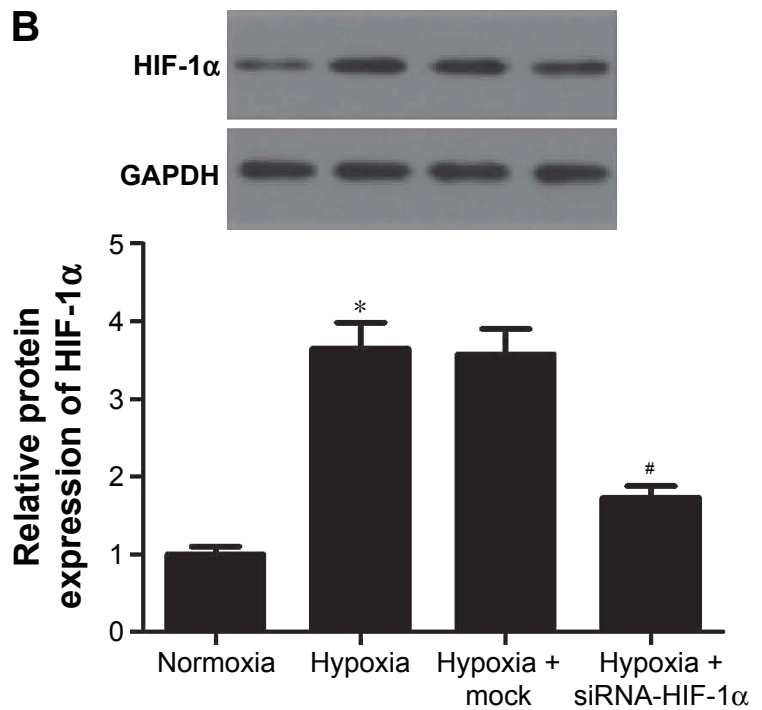

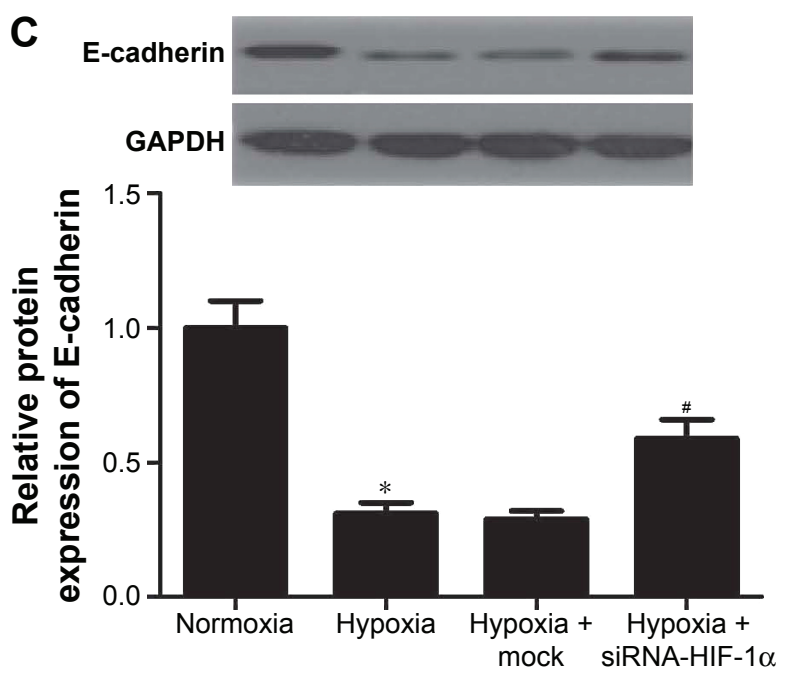

Figure $4 \mathrm{HIF}-\mathrm{I} \alpha$ is involved in the prevention of hypoxia-induced EMT by PF.

Notes: MDA-MB-23I cells grown under normoxia and hypoxia were treated with $25 \mu \mathrm{M}$ PF for 24 hours. (A) PF inhibited the hypoxia-induced expression of HIF-I $\alpha$. (B) Knockdown of HIF-I $\alpha$ inhibited hypoxia-induced HIF-I $\alpha$ expression. (C) Knockdown of HIF-I $\alpha$ restored hypoxia-induced reduction of E-cadherin expression. Each bar represents the mean $\pm S D$. The results were reproduced in three independent experiments. $* P<0.05$ vs normoxia group, ${ }^{*} P<0.05$ vs hypoxia group.

Abbreviations: PF, paeoniflorin; EMT, epithelial-mesenchymal transition; SD, standard deviation.

lung cancer A549 cells by blocking cell cycle progression in the $\mathrm{G}(0) / \mathrm{G}(1)$ phase..$^{21} \mathrm{PF}$ also inhibited the tumor invasion and metastasis in human hepatocellular carcinoma cells. ${ }^{22}$ Furthermore, hypoxia was shown to induce cell migration and invasion in many types of cancers. In the current study, we found that the inhibitory effect of PF on viability was significantly higher on MDA-MB-231 and MCF-7 cells grown under hypoxic than in the normoxic conditions. We also found that PF prevented the hypoxia-induced migration and invasion of MDA-MB-231 and MCF-7 cells.

The EMT is an important molecular step in cancer progression. Reduction or a loss of E-cadherin expression has a crucial role in tumor progression to invasive cancer and is also one of the well-established hallmarks of EMT. ${ }^{5,7,23}$
In addition, it has been reported that hypoxic microenvironment plays a key role in the progression of EMT. ${ }^{11,24,25}$ In the current study, we found that PF prevented the hypoxiainduced EMT in breast cancer cells. These results suggest that PF blocks the migration and invasion of breast cancer cells by repressing EMT under hypoxic conditions.

HIF-1 is a transcription factor that is critical for tumor adaptation to microenvironmental stimuli, and consists of HIF- $1 \alpha$ and HIF-1 $\beta$ subunits. It was reported that HIF- $1 \alpha$ was an important upstream regulator of the EMT process. In addition, hypoxia induces HIF- $1 \alpha$ expression, which induces the expression and activity of major transcription factors including TWIST, Snail, Slug, SIP1, and ZEB1, leading to the suppression of E-cadherin and induction of vimentin 
A

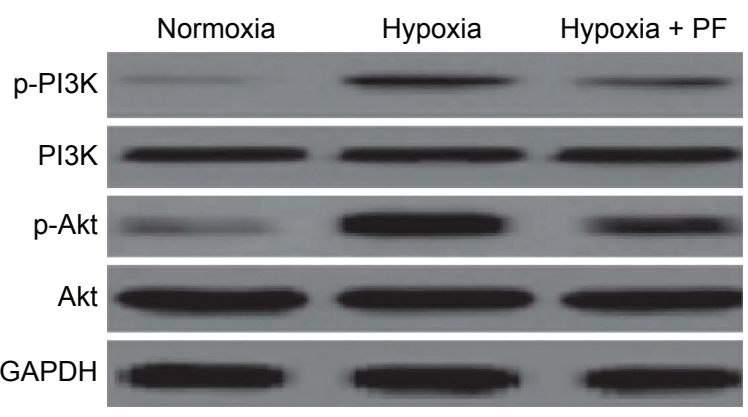

B

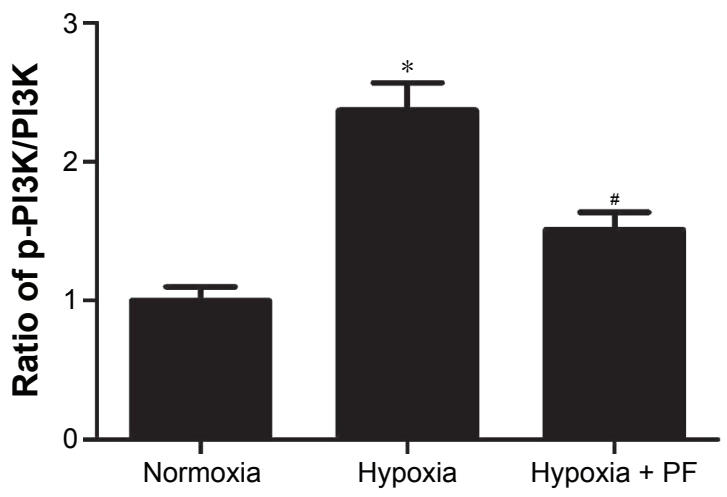

C

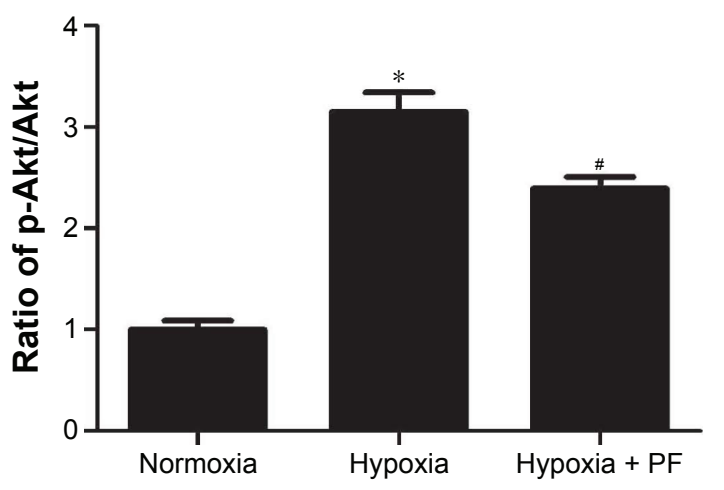

Figure 5 PF-mediated blockade of HIF-I $\alpha$ and EMT involves PI3K/Akt pathway.

Notes: MDA-MB-23I cells grown under normoxia or hypoxia were treated with $25 \mu$ M PF for 24 hours. (A) Western blot analysis to detect the protein expression levels of phosphorylated PI3K and Akt in different treated groups with the corresponding antibodies. (B, C) The relative protein expression levels of p-PI3K and p-Akt were quantified using Image-Pro Plus 6.0 software (Media Cybernetics, Silver Spring, MD, USA) and normalized to GAPDH. Each bar represents the mean \pm SD. The results were reproduced in three independent experiments. ${ }^{*} P<0.05$ vs normoxia group, ${ }^{\#} P<0.05$ vs hypoxia group.

Abbreviations: PF, paeoniflorin; EMT, epithelial-mesenchymal transition; SD, standard deviation.

in breast cancer cells. ${ }^{26-28}$ Consistent with these results, in the current study, we found that hypoxia-induced HIF-1 $\alpha$ expression and knockdown of HIF- $1 \alpha$ clearly inhibited hypoxia-induced E-cadherin expression; however, PF treatment suppressed the hypoxia-induced HIF-1 $\alpha$ expression. Our results suggest that PF prevented the hypoxia-induced EMT by inhibiting HIF-1 $\alpha$.

Previous studies have shown that PI3K/Akt signaling pathway is involved in HIF-1 $\alpha$ expression in tumor cells. ${ }^{29-31}$ For example, one study reported that a PI3K-specific inhibitor (LY294002) or overexpression of dominant-negative Akt significantly blunted HIF- $1 \alpha$ and NF- $\kappa \mathrm{B}$ induction in response to hypoxia in prostate cancer cells. ${ }^{32}$ Furthermore, PF inhibits human gastric carcinoma cell proliferation through upregulation of microRNA-124 and suppression of PI3K/Akt and STAT3 signaling. ${ }^{33}$ In the current study, we found that hypoxia treatment significantly increased the expression of phosphorylated PI3K and Akt in MDA-MB-231 cells. When treated with $\mathrm{PF}$, the increase in the levels of phosphorylated $\mathrm{PI} 3 \mathrm{~K}$ and Akt was reversed. These results suggest that PF prevented hypoxia-induced EMT in breast cancer cells by inhibiting HIF- $1 \alpha$ expression via modulation of PI3K/Akt signaling pathway.

\section{Conclusion}

In conclusion, PF prevented hypoxia-induced EMT in breast cancer cells by inhibiting HIF-1 $\alpha$ expression via modulation of PI3K/Akt signaling pathway. This finding provides evidence that PF can serve as a therapeutic agent for the treatment of breast cancer.

\section{Disclosure}

The authors report no conflicts of interest in this work.

\section{References}

1. DeSantis C, Ma J, Bryan L, Jemal A. Breast cancer statistics, 2013. CA Cancer J Clin. 2014;64(1):52-62.

2. Early Breast Cancer Trialists' Collaborative Group (EBCTCG). Aromatase inhibitors versus tamoxifen in early breast cancer: patient-level meta-analysis of the randomised trials. Lancet. 2015;386(10001): 1341-1352.

3. Early Breast Cancer Trialists' Collaborative Group (EBCTCG). Effects of chemotherapy and hormonal therapy for early breast cancer on recurrence and 15-year survival: an overview of the randomised trials. Lancet. 2005;365(9472):1687-1717.

4. Naume B, Synnestvedt M, Falk RS, et al. Clinical outcome with correlation to disseminated tumor cell (DTC) status after DTC-guided secondary adjuvant treatment with docetaxel in early breast cancer. J Clin Oncol. 2014;32(34):3848-3857.

5. Yang J, Weinberg RA. Epithelial-mesenchymal transition: at the crossroads of development and tumor metastasis. Dev Cell. 2008;14(6): $818-829$. 
6. Huber MA, Kraut N, Beug H. Molecular requirements for epithelialmesenchymal transition during tumor progression. Curr Opin Cell Biol. 2005;17(5):548-558.

7. Thiery JP. Epithelial-mesenchymal transitions in tumour progression. Nat Rev Cancer. 2002;2(6):442-454.

8. Rajaganeshan R, Prasad R, Guillou P, Poston G, Scott N, Jayne D. The role of hypoxia in recurrence following resection of Dukes' B colorectal cancer. Int J Colorectal Dis. 2008;23(11):1049-1055.

9. Lunt SJ, Chaudary N, Hill RP. The tumor microenvironment and metastatic disease. Clin Exp Metastasis. 2009;26(1):19-34.

10. Chen J, Imanaka N, Griffin J. Hypoxia potentiates Notch signaling in breast cancer leading to decreased E-cadherin expression and increased cell migration and invasion. Br J Cancer. 2010;102(2):351-360.

11. Jiang J, Tang YL, Liang XH. EMT: a new vision of hypoxia promoting cancer progression. Cancer Biol Ther. 2011;11(8):714-723.

12. Wu H, Wei W, Song L, Zhang L, Chen Y, Hu X. Paeoniflorin induced immune tolerance of mesenteric lymph node lymphocytes via enhancing beta 2-adrenergic receptor desensitization in rats with adjuvant arthritis. Int Immunopharmacol. 2007;7(5):662-673.

13. Zhang L-L, Wei W, Wang N-P, et al. Paeoniflorin suppresses inflammatory mediator production and regulates $\mathrm{G}$ protein-coupled signaling in fibroblast-like synoviocytes of collagen induced arthritic rats. Inflamm Res. 2008;57(8):388-395.

14. Nizamutdinova IT, Jin YC, Kim JS, et al. Paeonol and paeoniflorin, the main active principles of Paeonia albiflora, protect the heart from myocardial ischemia/reperfusion injury in rats. Planta Med. 2008; 74(1):14-18.

15. Zhang L, Zhang S. Modulating Bcl-2 family proteins and caspase-3 in induction of apoptosis by paeoniflorin in human cervical cancer cells. Phytother Res. 2011;25(10):1551-1557.

16. Wang $\mathrm{H}$, Zhou H, Wang C-X, et al. Paeoniflorin inhibits growth of human colorectal carcinoma HT 29 cells in vitro and in vivo. Food Chem Toxicol. 2012;50(5):1560-1567.

17. Wu H, Li W, Ge H-M, Liu P. Paeoniflorin induces the apoptosis of human gastric carcinoma cells by inhibiting activation of NF-kappa B. Acta Univ Med Nanjing. 2008;28(2):161-165.

18. Kapoor S. The emerging antineoplastic effects of paeoniflorin. Anticancer Drugs. 2013;24(4):429.

19. Jiang B-H, Jiang G, Zheng JZ, Lu Z, Hunter T, Vogt PK. Phosphatidylinositol 3-kinase signaling controls levels of hypoxia-inducible factor 1. Cell Growth Differ. 2001;12(7):363-369.

20. Zhong H, Chiles K, Feldser D, et al. Modulation of hypoxia-inducible factor $1 \alpha$ expression by the epidermal growth factor/phosphatidylinositol 3-kinase/PTEN/AKT/FRAP pathway in human prostate cancer cells: implications for tumor angiogenesis and therapeutics. Cancer Res. 2000;60(6):1541-1545.
21. Hung JY, Yang CJ, Tsai YM, Huang HW, Huang MS. Antiproliferative activity of paeoniflorin is through cell cycle arrest and the Fas/Fas ligand-mediated apoptotic pathway in human non-small cell lung cancer A549 cells. Clin Exp Pharmacol Physiol. 2008;35(2):141-147.

22. Lu J, He W, Song S, Wei W. Paeoniflorin inhibited the tumor invasion and metastasis in human hepatocellular carcinoma cells. Bratisl Lek Listy. 2013;115(7):427-433.

23. De Craene B, Berx G. Regulatory networks defining EMT during cancer initiation and progression. Nat Rev Cancer. 2013;13(2):97-110.

24. Marie-Egyptienne DT, Lohse I, Hill RP. Cancer stem cells, the epithelial to mesenchymal transition (EMT) and radioresistance: potential role of hypoxia. Cancer Lett. 2013;341(1):63-72.

25. Semenza GL. Hypoxia-inducible factors: mediators of cancer progression and targets for cancer therapy. Trends Pharmacol Sci. 2012; 33(4):207-214.

26. Zhou G, Dada LA, Wu M, et al. Hypoxia-induced alveolar epithelial-mesenchymal transition requires mitochondrial ROS and hypoxia-inducible factor 1. Am J Physiol Lung Cell Mol Physiol. 2009;297(6):L1120-L1130.

27. Salnikov AV, Liu L, Platen M, et al. Hypoxia induces EMT in low and highly aggressive pancreatic tumor cells but only cells with cancer stem cell characteristics acquire pronounced migratory potential. PLoS One. 2012;7:e46391.

28. Zhang L, Huang G, Li X, et al. Hypoxia induces epithelial-mesenchymal transition via activation of SNAI1 by hypoxia-inducible factor- $1 \alpha$ in hepatocellular carcinoma. BMC Cancer. 2013;13(1):108.

29. Gao N, Ding M, Zheng JZ, et al. Vanadate-induced expression of hypoxia-inducible factor $1 \alpha$ and vascular endothelial growth factor through phosphatidylinositol 3-kinase/Akt pathway and reactive oxygen species. J Biol Chem. 2002;277(35):31963-31971.

30. Zhong X-S, Zheng JZ, Reed E, Jiang B-H. SU5416 inhibited VEGF and HIF-1 $\alpha$ expression through the PI3K/AKT/p70S6K1 signaling pathway. Biochem Biophys Res Commun. 2004;324(2):471-480.

31. Kim C-H, Cho Y-S, Chun Y-S, Park J-W, Kim M-S. Early expression of myocardial HIF-1 $\alpha$ in response to mechanical stresses regulation by stretch-activated channels and the phosphatidylinositol 3-kinase signaling pathway. Circ Res. 2002;90(2):e25-e33.

32. Sun H, Liu Y, Huang Y, et al. YC-1 inhibits HIF-1 expression in prostate cancer cells: contribution of Akt/NF- $\mathrm{KB}$ signaling to HIF-1 $\alpha$ accumulation during hypoxia. Oncogene. 2007;26(27):3941-3951.

33. Zheng Y-B, Xiao G-C, Tong S-L, et al. Paeoniflorin inhibits human gastric carcinoma cell proliferation through up-regulation of microRNA-124 and suppression of PI3K/Akt and STAT3 signaling. World J Gastroenterol. 2015;21(23):7197-7207.
OncoTargets and Therapy

\section{Publish your work in this journal}

OncoTargets and Therapy is an international, peer-reviewed, open access journal focusing on the pathological basis of all cancers, potential targets for therapy and treatment protocols employed to improve the management of cancer patients. The journal also focuses on the impact of management programs and new therapeutic agents and protocols on
Dovepress

patient perspectives such as quality of life, adherence and satisfaction The manuscript management system is completely online and includes a very quick and fair peer-review system, which is all easy to use. Visit http://www.dovepress.com/testimonials.php to read real quotes from published authors. 